\title{
Capacidade para o trabalho de funcionários da prefeitura de um campus universitário público*
}

\author{
The working capacity of a public university employees
}

\section{Capacidad laboral de empleados municipales de un campus universitario público}

Aline Loiola Moura ${ }^{1}$, Lucia Margarete dos Reis ${ }^{2}$, Marli Terezinha Oliveira Vannuchi ${ }^{3}$, Maria do Carmo Lourenço Haddad ${ }^{4}$, Rita de Cássia Domansky ${ }^{5}$

\footnotetext{
* Trabalho de conclusão do curso de Residência em Gerência dos Serviços de Enfermagem da Universidade Estadual de Londrina.

${ }^{1}$ Enfermeira. Londrina, PR, Brasil. E-mail: aline.loiola@yahoo.com.br.

2 Enfermeira. Londrina, PR, Brasil. E-mail: luciamargarete@gmail.com.

${ }^{3}$ Enfermeira, Doutora em Saúde Pública. Professora Titular do Departamento de Enfermagem da Universidade Estadual de Londrina (UEL). Londrina, PR, Brasil. E-mail:vannuchi@sercomtel.com.br.

4 Enfermeira, Doutora em Enfermagem. Professora Associada do Departamento de Enfermagem da UEL. Londrina, PR, Brasil. E-mail: carmohaddad@gmail.com.

5 Enfermeira, Doutora em Enfermagem. Enfermeira da Divisão de Assistência à Saúde da Comunidade da UEL. Londrina, PR, Brasil. E-mail: r.domansky@sercomtel.com.br.
}

\section{RESUMO}

Estudo cujo objetivo foi verificar a capacidade para o trabalho entre trabalhadores da prefeitura de um campus universitário público. Pesquisa epidemiológica descritiva e quantitativa. A amostra foi composta por 307 trabalhadores, a maior parte dos funcionários era do sexo masculino; a faixa etária de maior prevalência foi 41 a 60 anos e evidenciou-se o envelhecimento da força de trabalho. Quanto à avaliação da capacidade para o trabalho, identificou-se que $58,9 \%$ dos entrevistados obtiveram um Índice de Capacidade para o Trabalho (ICT) bom; 37,8\%, moderado; e 3,2\%, baixo. Os entrevistados que referiram alguma lesão por acidente de trabalho ou alguma doença corresponderam a $84,3 \%$ da amostra; no entanto, $12,3 \%$ relataram que frequentemente, ou algumas vezes, precisam diminuir o ritmo de trabalho devido à presença de lesão ou doença. Considerando o exposto, o serviço de saúde do trabalhador deve estar atento a todos os trabalhadores, em especial quando se trata do ICT. Descritores: Avaliação da Capacidade de Trabalho; Envelhecimento; Saúde do Trabalhador; Enfermagem do Trabalho.

\section{ABSTRACT}

The objective of this study was to identify the working capacity of a public university employees. This is a descriptive and quantitative epidemiological study. The sample was comprised of 307 employees; most were male; of age between 41 and 60 years, showing that the workforce has aged. Regarding the evaluation of the Working Capacity Index (WKI), a fair WCI was found for $58.9 \%$ of the interviewees; moderate $\mathrm{WCI}$ for $37.8 \%$, and low $\mathrm{WCI}$ for $3.2 \%$. The interviewees who reported any occupational lesion or disease corresponded to $84.3 \%$ of the sample; however, $12.3 \%$ reported that often, or sometimes, they found it necessary to reduce their rhythm at work due to a lesion or disease. Hence, workers' health services must keep all workers at sight, especially considering their WCI.

Descriptors: Work Capacity Evaluation; Aging; Occupational Health; Occupational Health Nursing.

\section{RESUMEN}

Estudio que objetivó verificar la capacidad laboral entre trabajadores municipales de un campus universitario público. Investigación epidemiológica, descriptiva y cuantitativa. Muestra compuesta por 307 trabajadores. La mayoría era de sexo masculino; faja etaria prevalente de 41 a 60 años, evidenciándose el envejecimiento de la fuerza de trabajo. En cuanto a la evaluación de la capacidad laboral, se identificó que 58,9\% de los entrevistados obtuvieron un Índice de Capacidad para el Trabajo (ICT) bueno, 37,8\% moderado, y 3,2\% bajo. De los entrevistados, 84,3\% refirió alguna lesión por accidente laboral o bien alguna enfermedad; mientras que $12,3 \%$ informaron que con frecuencia o a veces, necesitan disminuir el ritmo de trabajo debido a la presencia de lesiones o enfermedades. Considerando lo expuesto, el servicio de salud del trabajador debe mantenerse atento a todos los trabajadores, en particular en lo referente al ICT.

Descriptores: Evaluación de Capacidad de Trabajo; Envejecimiento; Salud Laboral; Enfermería del Trabajo. 


\section{INTRODUÇÃO}

A capacidade para o trabalho é a base de bem estar dos indivíduos e está relacionada à competência que a pessoa tem para executar seus afazeres em função das exigências do trabalho, levando em consideração diversos fatores, como aspectos sociodemográficos, estilo de vida, processo de envelhecimento, organização e ambiente de trabalho(1-2).

É importante ressaltar que muitas profissões, além das exigências mentais, demandam também esforços físicos, como levantamento e transporte de peso, esforços repetitivos e repentinos, posturas inadequadas, inclinação simultânea, sobrecarga postural e do sistema músculo-esquelético, e necessitam de medidas de promoção à saúde, considerando a possibilidade de perda da capacidade para o trabalho(3).

Muitos trabalhadores em atividades e distantes da aposentadoria, já podem estar apresentando redução da capacidade para o trabalho, desencadeando respostas fisiológicas, psicológicas e comportamentais, com efeitos negativos sobre a saúde e a capacidade para o trabalho ${ }^{(4)}$.

Estudos realizados por pesquisadores do Instituto Finlandês de Saúde Ocupacional no período de 1981 a 1992, sobre capacidade para o trabalho e envelhecimento funcional, resultaram na elaboração do instrumento para avaliar o Índice de Capacidade para o Trabalho (ICT). No Brasil, as pesquisas sobre esse tema iniciaram-se após a adaptação e validação do ICT para a língua portuguesa realizada em $1997^{(1)}$.

Este instrumento permite avaliar a capacidade para o trabalho atual comparada com o melhor de toda a vida; a capacidade para o trabalho em relação às exigências físicas e mentais; número atual de doenças auto-referidas e as com diagnóstico médico; impedimento para o trabalho devido às doenças; faltas no trabalho por doenças nos últimos 12 meses; prognóstico próprio sobre a capacidade para o trabalho daqui a dois anos; capacidade de apreciar as atividades diárias, se sentir ativo e alerta, e com esperança para o futuro(1).

Problemas de saúde relacionados ao trabalho são comuns entre trabalhadores, e a capacidade рага o trabalho precisa ser preservada. Para que isso ocorra, o compromisso com a promoção à saúde deve acontecer em conjunto com outros setores, como as organizações governamentais e não governamentais, setores sociais, sindicatos, entre outros(5).
É importante priorizar a capacidade de trabalho em todas as fases ativas do trabalhador, pois determinados desempenhos laborais exigem atividade intensa associada ao desgaste físico e mental. Ressalta-se que a qualidade de vida durante a fase em que o indivíduo está inserido no trabalho, é importante para garantir à sociedade benefício futuro com menor custo para manter a saúde do idoso saudável.

Enquanto residente de gerência dos serviços de enfermagem da Universidade Estadual de Londrina-PR, ao realizar um estudo com os funcionários da prefeitura do Campus Universitário desta universidade, foi identificado o perfil sociodemográfico e ocupacional, evidenciou-se que o envelhecimento da força de trabalho é marcante, bem como é significativa a parcela de profissionais que atuam em cargos de auxiliares operacionais, ou seja, atividades como serviços de manutenção em geral, serviços de zeladoria, serviços de vigilância, serviços de carpintaria e jardinagem entre outros, que podem colocar em risco sua capacidade para o trabalho.

Considerando que esses trabalhadores são responsáveis pela manutenção e funcionamento de vários setores da instituição, bem como não foram desenvolvidas pesquisas no sentido de analisar minuciosamente esta força de trabalho, apesar de executarem atividades que potencialmente podem levar a um comprometimento da capacidade para o trabalho, o presente estudo teve como objetivo verificar a capacidade para o trabalho, em trabalhadores da prefeitura de um campus universitário público.

\section{METODOLOGIA}

Trata-se de um estudo de abordagem quantitativo do tipo descritivo-exploratório de corte transversal, realizado na Prefeitura do Campus Universitário da Universidade Estadual de Londrina (UEL).

A Prefeitura do Campus Universitário é o órgão que planeja, elabora, executa e controla os projetos relacionados com a estrutura física do campus, incluindo a programação e administração das áreas públicas, bem como todas as atividades de manutenção da Universidade ${ }^{(6)}$.

Nesse setor estão lotados 404 servidores e todos foram convidados a participar do estudo independente de idade, sexo e categoria profissional. Adotou-se como 
critérios de inclusão do sujeito no estudo, a aceitação e disponibilidade em responder o questionário. Não foram incluídos na pesquisa os funcionários que estavam em férias, licença prêmio, licença médica devido a doenças, acidentes ou outros motivos.

Devido à diversidade de ocupação verificada entre os trabalhadores, as mesmas foram agrupadas de acordo com suas funções laborativas, independentes de suas secretarias de origem, sendo classificadas em cargos administrativos, técnicos e função de auxiliar operacional.

Considerando o tamanho da população a ser pesquisada foi necessário contar com a colaboração de seis acadêmicos do curso de enfermagem da UEL. Os estudantes foram previamente capacitados para a coleta de dados, em três encontros para discussão sobre os objetivos do estudo, composição e estrutura do instrumento e etapas a serem cumpridas para a sua realização. Os entrevistadores receberam do pesquisador líder, que também coletou os dados, instruções detalhadas, inclusive escritas, sobre cada item que compõe o instrumento, e como realizar a abordagem do entrevistado no trabalho, incluindo a importância da obtenção de concordância do entrevistado em participar do estudo, com a assinatura do Termo de Consentimento Livre e Esclarecido (TCLE), em duas vias (ficando uma via com o entrevistado e a outra com o pesquisador). 0 período de coleta de dados se deu de março a junho de 2010.

A primeira parte do instrumento, elaborado por Domansky(7), é composta pela caracterização sociodemográfica e ocupacional da população em estudo.

Na segunda parte do instrumento, constam os dados referentes ao Índice de Capacidade para o Trabalho(1), composto por 60 questões às quais são atribuídas uma pontuação. O escore total varia de sete a 49 pontos, escores de sete a 27 pontos correspondem à baixa capacidade para o trabalho, de 28 a 36 pontos correspondem à moderada capacidade, de 37 a 43 pontos à boa capacidade, e finalmente, de 44 a 49 pontos à ótima capacidade (Quadro 1).

Quadro 1: Classificação da Capacidade para o Trabalho e objetivos das medidas segundo o escore alcançado.

\begin{tabular}{|c|c|c|}
\hline Escore alcançado & Classificação da Capacidade para o Trabalho & Objetivos das medidas \\
\hline 7 a 27 pontos & Baixa & Restaurar a capacidade para o trabalho \\
\hline 28 a 36 pontos & Moderada & Melhorar a capacidade para o trabalho \\
\hline 37 a 43 pontos & Boa & Apoiar a capacidade para o trabalho \\
\hline 44 a 49 pontos & Ótima & Manter a capacidade para o trabalho \\
\hline
\end{tabular}

Fonte: TUOMl; et al., 2005

Os dados coletados foram digitados e armazenados em banco de dados no formato EPI INFO 3.5.1. Рага cálculo dos dados utilizou-se a estatística descritiva.

O presente estudo obteve aprovação do Comitê de Ética em Pesquisa da UEL, sob o parecer no 147/2009, CAAE $n^{\circ}$ 012002680009. Todos os entrevistados assinaram o TCLE.

\section{RESULTADOS}

A população do estudo foi composta por 307 trabalhadores, os quais apresentaram as características sociodemográficas demonstradas na Tabela 1. 
Tabela 1: Distribuição das características sociodemográficas dos trabalhadores da prefeitura de um campus universitário público. Londrina, PR, 2010.

\begin{tabular}{|c|c|c|c|}
\hline Variáveis & Categoria & 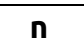 & $\%$ \\
\hline \multirow{4}{*}{ Sexo } & Masculino & 262 & 85,3 \\
\hline & Feminino & 45 & 14,7 \\
\hline & $<30$ & 22 & 7,2 \\
\hline & $31-40$ & 47 & 15,3 \\
\hline \multirow[t]{4}{*}{ Idade } & $41-50$ & 104 & 33,9 \\
\hline & $51-60$ & 100 & 32,6 \\
\hline & $>60$ & 34 & 11,1 \\
\hline & Solteiro & 40 & 13,0 \\
\hline \multirow{3}{*}{ Situação Conjugal } & União estável & 225 & 73,3 \\
\hline & Separado/divorciado & 33 & 10,7 \\
\hline & Viúvo & 9 & 2,9 \\
\hline \multirow{2}{*}{ Cor da Pelo } & Branca & 189 & 61,6 \\
\hline & Não branca & 118 & 38,4 \\
\hline \multirow{6}{*}{ Religião } & Sim & 293 & 95,4 \\
\hline & Não & 14 & 4,6 \\
\hline & Fundamental incompleto & 42 & 13,7 \\
\hline & Fundamental completo & 28 & 9,1 \\
\hline & Médio incompleto & 23 & 7,5 \\
\hline & Médio completo & 130 & 42,3 \\
\hline \multirow[t]{5}{*}{ Escolaridade } & Supletivo & 1 & 0,3 \\
\hline & Superior incompleto & 41 & 14,7 \\
\hline & Superior completo & 21 & 6,8 \\
\hline & Stricto senso & 5 & 1,6 \\
\hline & Especialização & 12 & 3,9 \\
\hline
\end{tabular}

A distribuição dos servidores segundo o ICT é "moderada" e 3,2\% como "baixa" capacidade para o demonstrado na Tabela 2. Dos entrevistados, 58,9\% trabalho. obteve classificação do ICT como "boa", 37,8\%

Tabela 2: Distribuição dos trabalhadores da Prefeitura de um campus universitário público segundo Índice de capacidade para o trabalho. Londrina, PR, 2010.

\begin{tabular}{ccc}
\hline Capacidade para o Trabalho & $\mathbf{n}$ & \% \\
\hline Baixa (7-27) & 10 & 3,2 \\
Moderada (28-36) & 116 & 37,8 \\
Boa (37-43) & 181 & 58,9 \\
\hline Total & $\mathbf{3 0 7}$ & $\mathbf{1 0 0}$ \\
\hline
\end{tabular}

A Tabela 3 demonstra a distribuição dos trabalhadores segundo índice de capacidade para o trabalho e faixa etária. 
Tabela 3: Distribuição dos trabalhadores da Prefeitura de um campus universitário público segundo a Faixa Etária. Londrina, PR, 2010.

\begin{tabular}{lllllllll}
\hline ICT/Faixa etária & \multicolumn{2}{c}{$<\mathbf{3 0}$ anos } & \multicolumn{2}{c}{$\mathbf{3 1 - 4 0}$ anos } & \multicolumn{2}{c}{$\mathbf{4 1 - 6 0}$ anos } & \multicolumn{2}{c}{$\mathbf{6 0}$ anos } \\
& $\mathbf{n}$ & $\mathbf{\%}$ & $\mathbf{n}$ & $\mathbf{\%}$ & $\mathbf{n}$ & $\mathbf{\%}$ & $\mathbf{n}$ & $\mathbf{\%}$ \\
\hline Baixa & - & - & 1 & 2,1 & 9 & 4,4 & - & - \\
Moderada & 10 & 45,5 & 16 & 34,0 & 72 & 35,3 & 18 & 52,9 \\
Boa & 12 & 54,5 & 30 & 63,8 & 123 & 60,6 & 16 & 47,1 \\
\hline Total & $\mathbf{2 2}$ & $\mathbf{1 0 0}$ & $\mathbf{4 7}$ & $\mathbf{1 0 0}$ & $\mathbf{2 0 4}$ & $\mathbf{1 0 0}$ & $\mathbf{3 4}$ & $\mathbf{1 0 0}$ \\
\hline
\end{tabular}

Observou-se que não houve diferenças significativas na classificação do ICT relacionado à idade dos entrevistados.

Dos trabalhadores $71,9 \%$ exerciam função de auxiliar operacional, $14,9 \%$ ocupavam cargos técnicos, e $13 \%$ ocupavam cargos administrativos.

Em uma escala de zero a 10 para auto-avaliação da capacidade para o trabalho, $24,7 \%(n=76)$ dos servidores atribuíram o valor máximo, $35,8 \%(n=110)$ se avaliaram com nota nove, seguidos por $31,5 \%(n=97)$ com o valor oito, $5,8 \%(n=18)$ com valor sete, $0,9 \%(n=3)$ entrevistados conferiram o valor seis para sua capacidade atual ao trabalho, sendo que $0,9 \% \quad(n=3)$ dos trabalhadores atribuíram o valor cinco, quatro e três.

Em relação à lesão por acidente de trabalho ou alguma doença, $84,3 \%$ ( $n=259)$ relataram que possuem alguma doença ou lesão por acidente de trabalho, destes $60,5 \%$ ( $n=186)$ referiram que mesmo possuindo alguma lesão ou doença, não há impedimento para o trabalho.

\section{DISCUSSÃO}

A caracterização sociodemográfica mostra que a população do estudo é predominantemente masculina (85,3\%). Quanto à faixa etária, observou-se que a idade variou de 20 a 69 anos. A média das idades foi de 48 anos com desvio padrão de 10 anos o que determina um coeficiente de variação de $21 \%$, identificando que o conjunto das idades apresenta uma homogeneidade média. Houve uma prevalência de indivíduos com mais de 41 anos (77,6\%) dados que corroboram com achados num estudo sobre capacidade para o trabalho entre trabalhadores de higiene e limpeza de um hospital universitário público, em que a maioria da população também possuía mais de 41 anos de idade ${ }^{(8)}$.

O envelhecimento da população trabalhadora é um tema frequente nas sociedades modernas, e a velocidade do envelhecimento populacional brasileiro será ainda maior, tendo em vista que a diminuição das taxas de natalidade e mortalidade tem colaborado para o aumento de pessoas idosas e em idade produtiva. Desta forma fazse necessário a busca por estratégias para manterem boas condições de saúde, capacidade para o trabalho, autonomia e integração social dos trabalhadores em processo de envelhecimento( ${ }^{(9)}$.

Quanto ao estado civil, 73,3\% da população relatou possuir união estável, resultado semelhante ao de uma pesquisa sobre fatores associados à capacidade para o trabalho de trabalhadores do Setor Elétrico, onde se identificou que $75,2 \%$ também possuíam união estável(4) e que esta condição pode favorecer uma boa capacidade para o trabalho.

Quanto à religiosidade, 95,4\% dos trabalhadores declararam que possuem religião. Considerando que a população do estudo está em fase de envelhecimento pode-se associar com estudos que têm demonstrado que as pessoas idosas são mais fortemente religiosas do que as mais jovens ${ }^{(10)}$.

Em relação à escolaridade, 42,3\% dos entrevistados completaram o ensino médio, 6,8\% possuíam ensino superior completo, 3,9\% especialização e 1,6\% possuíam especialização a nível Stricto Senso. Observa-se que o maior percentual desta população possui no mínimo ensino médio completo. De acordo com estudo esse é um fator facilitador para as ações de educação em saúde e fator protetor para déficits $\operatorname{cognitivos}^{(11)}$, de modo a facilitar para o indivíduo a busca por hábitos de vida mais saudáveis.

Os resultados demonstraram que $41,0 \%$ da população pesquisada apresentaram capacidade para o trabalho nas categorias "moderada" e "baixa" e os demais referiram possuir "boa" capacidade, resultado que contraria estudos que afirmam que a diminuição da capacidade para o trabalho está relacionada ao fator idade avançada ${ }^{(1,4-5)}$. Isto pode estar relacionado ao fato de que o próprio trabalhador entrevistado pode se sentir intimidado ao responder sinceramente o questionário, e 
também podendo ser justificado pela dificuldade na compreensão das perguntas e das respostas, o que caracteriza uma limitação deste estudo.

Em relação à faixa etária, os entrevistados de 20 a 40 anos em sua maioria apresentaram uma classificação para o ICT "boa" que corresponde a 60,8\%, 37,6\% "moderado", $1,6 \%$ apresentaram "baixa" capacidade. Nos trabalhadores acima de 41 anos, a maior prevalência concentrou-se no ICT classificado como "bom", resultado que contradiz estudos que afirmam que quanto maior a idade, menor o ICT ${ }^{(1,5,12)}$, sendo que nenhum dos servidores dentro desta faixa etária considerou sua capacidade para o trabalho "baixa".

A questão do envelhecimento relacionado ao trabalho é um tema preocupante, estudo de reflexão sobre envelhecimento e trabalho afirma que modificações em vários sistemas do corpo humano levam a diminuição gradativa na eficácia de cada um deles, considerando que o envelhecimento causado pelo trabalho antecipa estágios do ciclo de vida do homem, muitas vezes não respeitando o que seria apropriado nesse processo(13). Informação que contradiz com o resultado desta pesquisa, visto que não houve diferenças significativas na classificação do ICT relacionado à idade dos entrevistados.

Quanto à área de ocupação auto-referida por trabalhadores da instituição em estudo, observou-se que $71,9 \%$ exercem função de auxiliar operacional, 14,9\% ocupam cargos técnicos, e $13 \%$ ocupam cargos administrativos.

Observa-se que o caráter de trabalho realizado pela maioria da população desta pesquisa, de acordo com um estudo sobre prevalência de sintomas osteomusculares e fatores associados em trabalhadores de uma indústria metalúrgica de Canoas-RS, pode levá-los a problemas de saúde relacionados aos seus afazeres como doenças osteomusculares, podendo assim, prejudicar sua capacidade laboral ${ }^{(14)}$.

Parcela significativa destes servidores foi classificada na função de auxiliar operacional, como os motoristas, seguranças, zeladores, jardineiros, auxiliares de manutenção, entre outros. Desta forma, pode-se afirmar que muitos lidam em suas atividades diárias com levantamento de pesos, longas jornadas em pé, uso de equipamentos não seguros, exposição ao calor e frio excessivos, umidade, sendo que, os problemas que podem causar para a saúde são inúmeros ${ }^{(14)}$.

Dos entrevistados que atuam em atividades de auxiliar operacional, 54,3\% apresentaram ICT classificado como "boa", 41,6\% "moderada" e 4,1\% "baixa" capacidade, sendo que dos trabalhadores que ocupam cargos técnicos $21,7 \%$ classificaram sua capacidade como "moderada" e 78,3\% "boa", dos ocupantes de cargos administrativos 62,5\% apresentaram ICT "boa", 35,0\% "moderada" e 2,5\% classificaram como "baixa".

Mais uma vez os resultados desta pesquisa contestam estudos que afirmam que características de trabalhos que exigem esforços físicos estão relacionados à baixa capacidade para o trabalho, fato que pode ser explicado pela intimidação ou não aceitação dos trabalhadores em relação a sua real capacidade laboral $(1,4-5,14)$.

Ao serem questionados quanto a sua capacidade para o trabalho atual, em uma escala de zero a $10,24,7 \%(n=76)$ dos servidores atribuíram o valor máximo, 35,8\% ( $n=110)$ se avaliaram com nota nove, seguidos por $31,5 \%(n=97)$ com o valor oito, $5,8 \%(n=18)$ com valor sete, $0,9 \%(n=3)$ entrevistados conferiram o valor seis para sua capacidade atual ao trabalho, sendo que $0,9 \% \quad(n=3)$ dos trabalhadores atribuíram o valor cinco, quatro e três. Nenhum se auto-avaliou com valor de zero a dois. Observa-se que a maior parte da população considera seu ICT adequado, fato que pode estar associado com sua satisfação no trabalho.

Em um estudo sobre satisfação e capacidade para o trabalho entre docentes universitários houve uma correlação entre satisfação e ICT entre os docentes da área da saúde, indicando que quanto mais satisfeitos estiverem, maior será o seu índice de capacidade para o trabalho, desta forma, a satisfação é um fator essencial para os trabalhadores desempenharem suas tarefas de maneira eficaz ${ }^{(15)}$.

A população do estudo foi questionada se possuía alguma lesão por acidente de trabalho ou alguma doença, $84,3 \%$ ( $n=259$ ) relataram que possuem alguma doença ou lesão por acidente de trabalho, destes 60,5\% ( $n=186)$ referiram que mesmo possuindo alguma lesão ou doença, não há impedimento para o trabalho. Evento que pode ser explicado devido ao fato da maioria dos entrevistados serem do sexo masculino, pois, de acordo com o Ministério da Saúde, os homens em geral têm dificuldade em reconhecer suas necessidades, cultivando o 
pensamento mágico que rejeita a possibilidade de adoecer ou prejudicar sua capacidade para o trabalho(16).

A saúde é entendida como um ocasionador relevante da capacidade para o trabalho. Desta forma, quanto melhor o estado de saúde dos indivíduos, melhor a capacidade para o trabalho, independente das características demográficas e ocupacionais ${ }^{(1,4-5)}$.

Em relação aos sintomas causados pelas doenças, $10,7 \%$ ( $n=33$ ) afirmaram ser capazes de fazer seu trabalho, mas o mesmo causa alguns sintomas, 9,4\% $(n=29)$ relataram que algumas vezes precisam diminuir seu ritmo de trabalho ou diminuir métodos de trabalho por conta da doença ou lesão por acidente, $2,9 \% \quad(n=9)$ referiram que frequentemente precisam diminuir o ritmo do trabalho ou diminuir métodos de trabalho.

A análise desse aspecto ainda pode ser entendida pelo fato do sexo masculino ser predominante nesse estudo, pois pesquisas que relacionam a capacidade para o trabalho entre indivíduos do sexo masculino com o sexo feminino, demonstram que os homens manifestam melhor capacidade para o trabalho quando comparada às mulheres, assim os problemas relacionados a saúde tendem a não se manifestar a ponto de impedirem o processo de trabalho ${ }^{(1,3,5)}$.

Dos entrevistados que relataram que frequentemente, ou algumas vezes precisam diminuir o ritmo de trabalho devido à presença de lesão ou doença, corresponde a $12,3 \%$. Destes, $78,9 \%$ apresentou uma capacidade moderada para o trabalho e $18,4 \%$ um ICT baixo.

A capacidade para o trabalho diz respeito às qualificações do trabalhador para lidar com as exigências do trabalho, sendo baseada em sua capacidade física, mental e social. A capacidade para o trabalho, considerada como resultante de um processo dinâmico entre recursos do indivíduo em relação ao seu trabalho, sofre modificações em função de vários fatores, entre eles a condição de saúde, as características sociodemográficas, o estilo de vida, o envelhecimento e os fatores relacionados ao trabalho(1-4).

\section{CONCLUSÃO}

Entre os aspectos sociodemográfico dos funcionários da prefeitura de um campus universitário público, concluiu-se que a masculinização nesta força de trabalho é evidente, destacando-se o envelhecimento dos trabalhadores, sendo que $77,6 \%$ apresentam mais de 41 anos de idade, e que as atividades desenvolvidas por significativa parcela da população, são atividades que requerem esforço físico, podendo levar o funcionário a perda precoce de sua capacidade funcional.

A avaliação da capacidade para o trabalho identificou que $58,9 \%$ dos entrevistados obtiveram um ICT bom, $37,8 \%$ moderado e 3,2\% baixo. Desta forma, observou-se que os resultados desta pesquisa contradizem vários estudos que afirmam que a capacidade para o trabalho está relacionada ao fator idade e atividades que demandam esforço físico. Assim, as medidas a serem realizadas deverão apoiar a capacidade para o trabalho.

Evidenciou-se que uma parcela significativa dos entrevistados referiu possuir alguma lesão por acidente de trabalho ou alguma doença, o que corresponde a $84,3 \%$. No entanto, $12,3 \%$ relataram que frequentemente, ou algumas vezes, precisam diminuir o ritmo de trabalho devido à presença de lesão ou doença.

Ficou evidente que os fatores relacionados à capacidade para o trabalho são diversos, desta forma, o Serviço de Saúde do Trabalhador da instituição em que o estudo foi realizado deve implantar ações de prevenção e recuperação aos trabalhadores com risco à diminuição da capacidade funcional, garantindo um momento para reflexão sobre o processo de trabalho, por meio de discussões em grupos com profissionais especializados em saúde do trabalhador.

Como limitação do estudo, destaca-se a escassez de pesquisas sobre capacidade para o trabalho em população cujas funções são variadas, encontrando somente estudos de população pertencentes a uma única categoria profissional. Isso de certa forma, dificulta o processo de cotejamento entre a literatura especializada e os dados encontrados nesta pesquisa. Nesse sentido, recomenda-se que novos estudos sejam realizados sobre a capacidade para o trabalho entre trabalhadores atuantes em instituições de ensino, independente de suas funções.

Assim, ressalta-se que a melhoria da capacidade funcional já praticada enquanto o trabalhador estiver trabalhando poderá contribuir na qualidade de vida na aposentadoria desses trabalhadores e com a redução dos custos para manter a saúde do idoso. 


\section{REFERÊNCIAS}

1. Tuomi K, Ilmarinen J, Jahkola A, Katajarinne L, Tulkki A. Índice de capacidade para o trabalho. Tradução de Fischer F.M. (coord.). São Carlos: Editora EdUFSCar, 2005.59 p.

2. Renosto A, Biz P, Hennington EA, Pattussi MP. Confiabilidade teste-reteste do Índice de Capacidade para o Trabalho em trabalhadores metalúrgicos do Sul do Brasil. Rev Bras Epidemiol. 2009;12(2):217-225.

3. Walsh IAP, Corral S, Franco RN, Canetti EEF, Alem MER, Coury HJCG. Capacidade para o trabalho em indivíduos com lesões músculo-esqueléticas crônicas. Rev Saúde Pública. 2004;38(2):149-156.

4. Martinez MC, Latorre MRDO. Fatores associados à capacidade para o trabalho de trabalhadores do Setor Elétrico. Cad. Saúde Pública. 2009;25(4):761-72.

5. Andrade CB, Monteiro MI. Envelhecimento e capacidade para o trabalho dos trabalhadores de higiene e limpeza hospitalar. Rev. esc. enferm. USP. 2007;41(2):237-44.

6. Reitoria da Universidade Estadual de Londrina - PR 2012. Available from: http://www.uel.br/pcu/portal/pages/diretoriade-servicos---ds.php

7. Domansky RC. Adaptação transcultural e validação do instrumento "Bowel Function in the Community" para a língua portuguesa [dissertation]. São Paulo: Escola de Enfermagem/USP; 2004. 107 p.

8. Silva LG, Haddad MCL, Domansky RC, Vituri DW. Capacidade para o trabalho entre trabalhadores de higiene e limpeza de um hospital universitário público. Rev. Eletr. Enf. [Internet].2010;12(1):158-63. Available from: http://www.fen.ufg.br/revista/v12/n1/v12n1a19.htm. 9. Sampaio RF, Augusto VG. Envelhecimento e Trabalho: Um desafio para a agenda de reabilitação. Ver Bras Fisioter. 2012;16(2):94-101.

10. Farias RG, Santos SMA. Influência dos determinantes do envelhecimento ativo entre idosos mais idosos. Texto contexto - enferm., Florianópolis, v. 21, n. 1, Mar. 2012. Available from <http://www.scielo.br/scielo.php?script=sci_arttext\&pid=S010407072012000100019\&lng=en\&nrm=iso>.

11. Carlos, CALV, M. Sc. Análise do envelhecimento funcional precoce em funcionários de lavanderia terceirizada [dissertação]. [Belo Horizonte]. Universidade Federal de Viçosa. 2008. 87 p.

12. Salgado SML, Mafra SCT, Doimo LAD, Loreto MDS.

Percepção da capacidade para o trabalho e incidência de dores versus envelhecimento funcional precoce de auxiliares de agropecuária da Universidade Federal de Viçosa - MG. Oikos: revista brasileira de economia doméstica. 2011;22(2):108-30.

13. Souza RF, Matias HA, Brêtas ACP. Reflexões sobre envelhecimento e trabalho. Ver. Ciência \& Saúde Coletiva. 2010;15(6):2835-2843.

14. Picoloto, D, Silveira E. Prevalência de sintomas osteomusculares e fatores associados em trabalhadores de uma indústria metalúrgica de Canoas - RS. Ciênc. saúde coletiva. 2008;13(2):507-16.

15. Marqueze EC, Moreno CRC. Satisfação no trabalho e capacidade para o trabalho entre docentes Universitários. Psicologia em Estudo. 2009;14(1):7582.

16. Brasil. MS. Secretaria de atenção à saúde departamento de ações programáticas estratégicas. Política Nacional de Ação Integral à Saúde do Homem. Brasília, 2008.

Artigo recebido em 18/03/2011.

Aprovado para publicação em 09/08/2012.

Artigo publicado em 31/03/2013. 